\title{
Impact of climate anomalies on the functionality of beech trees in a mixed forest in the Italian south-eastern Alps
}

\author{
Luca Belelli Marchesini ${ }^{1,3}$, Riccardo Valentini ${ }^{2,3}$, Lorenzo Frizzera ${ }^{1}$, Mauro Cavagna ${ }^{1}$, Isaac Chini ${ }^{1}$, \\ Roberto Zampedri ${ }^{1}$, and Damiano Gianelle ${ }^{1}$ \\ ${ }^{1}$ Department of Sustainable Agro-Ecosystems and Bioresources, Research and Innovation Centre, Fondazione Edmund \\ Mach, San Michele all'Adige, Italy (luca.belellimarchesini@fmach.it) \\ ${ }^{2}$ Department for Innovation in Biological, Agro-Food and Forest Systems, University of Tuscia, Viterbo, Italy. \\ ${ }^{3}$ Department of Landscape Design and Sustainable Ecosystems, AgrarianDTechnological Institute, RUDN University, \\ Moscow, Russia.
}

The increased frequency and severity of extremes climatic events determined by the current and predicted scenarios of global climate changes have a large potential impact on the functionality of forest ecosystems and on their capacity of providing ecosystem services. These include climate warming mitigation capacity of forests which is exerted through carbon sequestration, carbon storage and the regulation of the energy balance by allocating incoming solar energy into transpiration rather than thermal energy. Assessing the size of the effects of increasing atmospheric temperature and climate anomalies on the functionality of forests, both in the short and mid-term, as well as their resilience capacity, is therefore of utmost importance in ecological research. European beech (Fagus sylvatica L.) forests, extensively represented over the continent, are known to be particularly vulnerable to late frosts, which limit their distribution into continental areas, and droughts, especially in the southernmost area of their geographical distribution.

The object of this study is an alpine mixed forest at the site of Cembra $(46.20 \mathrm{~N} ; 11.12 \mathrm{E}, 1250 \mathrm{~m}$ a.s.l) in the Trentino province (Italy). In May 2019 the forest experienced a late spring frost during the early development stage of beech leaves with resulting damage to canopies differing remarkably among individual plants. The ecological monitoring of beech trees started in June 2019 and has been since then carried out by means of clusters of traditional and Internet of Things based devices (Valentini et al. 2019) recording trees radial growth, sap flow density and characterizing the forest microclimatic space. The adopted experimental design consisted in the formation of two groups of trees $(n=18)$ featuring contrasting damaged/undamaged canopies, all selected from the dominant or subdominant layer of the forest canopy structure.

Here we present the rates and the seasonal patterns of beech trees stem radial growth and transpiration from June to November 2019, highlighting the differences among plants directly dependent on the damage conditions and interpreting the total observed tree level variability in relation to microclimate, surrounding forest structure and microtopography. The functional recovery dynamics of transpiration and stem growth in damaged plants will be addressed as an initial evaluation of short-term resilience capacity of the beech forest . 
